\title{
Angular momentum transfer in oblique impacts: Implications for 1989ML
}

\author{
Masahisa Yanagisawa and Sunao Hasegawa* \\ University of Electro-Communications, 1-5-1 Chofugaoka, Chofu-shi, Tokyo 182-8585, Japan
}

(Received October 10, 1998; Revised August 6, 1999; Accepted September 23, 1999)

\begin{abstract}
We conducted 10 shots of high-velocity oblique impact experiments $(1.95-3.52 \mathrm{~km} / \mathrm{s})$ using nylon projectiles and spherical mortar targets. Large craters were formed, but these targets were not disrupted by the impacts. We then calculated the efficiencies of momentum transfer from the projectile to the post-impact target for each experiment. The efficiencies of angular momentum transfer from the translational motion of the projectiles to the rotation of the post-impact targets were also derived. A representative efficiency of angular momentum transfer was calculated to be 0.17 for random successive collisions. The efficiency was applied to an equation expressing the precession angle of asteroids. It is shown that 1989ML, target of Japan-US asteroid-sample-return-mission (MUSES-C) would be tumbling.
\end{abstract}

\section{Introduction}

Collisions between asteroids must have played important roles in their rotational evolution (Davis et al., 1989). Spins of fragments from collisional catastrophic disruption of asteroidal parent bodies may dominate the present rotational states of asteroids. All asteroids, on the other hand, have also suffered many impacts that do not shatter them. In a collision without disruption, where the mass of the largest outcome is more than one-half the pre-impact body, a portion of the angular momentum of the translational motion of a projectile is transferred to the rotational angular momentum of the target asteroid (the largest outcome). The angular momentum of each asteroid is considered to accumulate quadratically as a random walk process through successive non-disruptive collisions. Based on these considerations, theoretical models of asteroidal rotational evolution have been developed in the last two decades (e.g., Harris, 1979; Dobrovolskis and Burns, 1984; Davis et al., 1989; Binzel, 1992).

The brightness of asteroids varies with their rotation because of their non-spherical shape, and the rotational states of asteroids are derived from the observation of their lightcurves. The lightcurves of some asteroids shows double periods. They may be attributed to forced-precession due to gravitational torque of their possible satellites (Binzel, 1985; Prokof'eva et al., 1995). Collision, on the other hand, changes the rotational state of asteroids and excites their freeprecession, while internal energy dissipation aligns rotational axis with body axes. Burns and Safronov (1973), hereafter B\&S1973, estimated the time scales of the excitation and the dissipation, and suggested the possibility of free-precession of small asteroids.

*Present address: Institute of Space and Astronautical Science, Sagamihara-shi, Kanagawa 229-8510, Japan.

Copy right(C) The Society of Geomagnetism and Earth, Planetary and Space Sciences (SGEPSS); The Seismological Society of Japan; The Volcanological Society of Japan; The Geodetic Society of Japan; The Japanese Society for Planetary Sciences.
It is therefore important to study the angular momentum transfer for non-disruptive collision in laboratory experiments. We do not still well understand the interior structure and the material of asteroids. The size of targets and projectiles is quite different between the asteroidal collision and the experiments, and we have not yet fully-reliable scaling laws to apply laboratory results to asteroids. It is difficult to simulate gravity effect in laboratory. Some of these problems may be solved by numerical simulation (Asphaug et al., 1996, 1998; Asphaug and Scheeres, 1999). The impact experiments are however the major source of our understanding on the phenomena at present.

Fujiwara and Tsukamoto's (1981) laboratory impact experiments with basaltic targets have shown that $10 \%$ of the orbital angular momentum is transferred to the rotation in the non-disruptive collisions. Yanagisawa et al. (1991) first conducted a series of experiments dedicated to obtain the fraction, and discussed the dependences of the fraction on impact angle and the hardness of target material. The impact velocity (less than $1 \mathrm{~km} / \mathrm{s}$ ) and the projectile material (lead and aluminum) in their experiments however are not typical for asteroidal collisions. Shirono et al. (1993) made 10 shots into spherical mortar targets with various impact velocities at fixed impact angle. The fraction transferred to the rotation varies from 0.04 to 0.4 , and seems to be independent of the impact velocity, $v$, at $v>2 \mathrm{~km} / \mathrm{s}$.

On the other hand, it has been proved that the fraction of the momentum transferred in the component tangential to target's surface is identical to the fraction of the angular momentum transferred in small cratering impact on spherical targets (Yanagisawa et al., 1996). The momentum of targets in horizontal component was measured in oblique impacts into horizontal surface of sand by Davis and Weidenschilling (1982), Gault and Schultz (1986), and Schultz and Gault (1986). The momentum of targets is less than $0.5(0.1-0.2$ in most cases) the projectiles' momentum in this component. The fraction of momentum transferred in this component was 
also studied by Yanagisawa et al. (1996) for various target materials such as mortar, iron, and aluminum. It varies from 0.2 to 0.5 for their mortar targets.

The number of experiments with spherical targets is yet very small at high impact velocity (e.g., $v \geq 2 \mathrm{~km} / \mathrm{s}$ ). In this paper, we measure both the momentum vector and the angular momentum of spherical target in the high-velocity impact, and obtained the efficiency of angular momentum transfer. Then, the effect of non-disruptive collisions on the rotation of 1989ML is discussed. This Near-Earth-Asteroid (NEA) is a target of Japan-US asteroid-sample-return-mission (MUSES-C) scheduled to be launched in 2002 and arrive at the asteroids in 2003 (Fujiwara et al., 1999).

\section{Experiments}

Projectiles are nylon spheres of $7 \mathrm{~mm}$ in diameter and $0.213 \mathrm{~g}$ in mass. Targets are made of mortar that was used in Yanagisawa et al. (1996) as Mortar-B. Its mechanical properties are listed there. We filled mixture of mortar powder and water into plastic balls each of which consists of two hemispherical shells, and made it to solidify. The shells were then removed and the spherical targets were allowed to dry completely. We tried to make them uniform and without any void, but some heterogeneity and void were inevitable. Imperfect fitting of the two shells caused a little deviation of the targets' shape from sphere. The largest targets were distorted a little due to the insufficient stiffness of the plastic balls. Each target has a hook to be supported by wire, and pins glued to it to facilitate an accurate measurement of the rotation rate. The diameter of the targets ranges between 60 and $150 \mathrm{~mm}$.

Glass Fiber Reinforced Plastic (GFRP) tape was wound around the equator of the targets, one of the poles of which projectiles hit. We tried to simulate qualitatively the effect of self-gravity with the tape, which prevents slow fragments from dispersing. The gravitational re-accumulation of fragments has been suggested to form asteroids of rubble-pile structure (Davis et al., 1989). The gravity may work in this way even in small asteroids while cratering and fragmentation are dominated by targets' strength. The angular momentum of a rubble-pile asteroid is the sum of the orbital and rotational angular momentum of the slow fragments. Preventing the fragments separating each other in the portion distant from the impact point may simulate such phenomena.

The other purpose of using the tape is to prevent the collisional disruption of targets whose surface curvature radius is small. We attempted to examine the effect of the curvature radius on the momentum and angular momentum transfer in the cratering impacts. Spherical targets of small curvature radius (e.g., $30 \mathrm{~mm}$ ) are too small to avoid collisional disruption without the tapes.

Projectiles were accelerated by a two-stage light gas gun. The trajectory of the projectiles is horizontal. A free fall system made the targets begin free fall less than a few tens of millisecond before impact. The post-impact target motion is thus free from the reaction of the supporting wire. Impact phenomena and the motion of the targets were recorded at 500 frames per second by a high-speed video movie camera (nac HSV-1000). Laser beam passing through gun-barrel was aimed at the targets when we set them up. It is not how- ever guaranteed that projectile's trajectory accurately coincides with the beam. Fortunately, we could record point-like flashes at impact points in two experiments (Shot Nos. L-54 and 55). We assumed that the trajectory of the projectiles was fixed in the recorded frames, and estimated the impact points for the other experiments. Each frame of the highspeed TV consists of 131 horizontal scanning lines. We assumed the standard deviation of the trajectory in vertical direction corresponding to the separation between the adjacent TV scanning lines. No deviation of the trajectory in the direction perpendicular to the projected frame plane is presumed. Projectile's incident angle, $\theta$, measured from surface normal at the impact point and its error are thus derived.

Because the post-impact targets move slowly, they receive blow of propellant gas of the gun. We made some experiments with targets of thin plastic disks that were fired without projectile, or the disks with small central hole through which projectiles pass. We measured the momenta of these targets divided by their cross section and calculated their average. Corrections for the momentum received from the gas, $\boldsymbol{p}_{g}$, were accordingly made by using to the following formula;

$$
\boldsymbol{p}_{g}=(10 \pm 3)\left(\mathrm{Ns} / \mathrm{m}^{2}\right) \cdot\left(\pi a^{2}\right) \frac{\boldsymbol{p}}{|\boldsymbol{p}|}
$$

The momentum vector of projectile and the radius of the targets are represented respectively by $\boldsymbol{p}$ and $a$. The correction amounts to 6 to $44 \%$ of the observed targets' momentum in the direction parallel to the projectiles' trajectory. Gun-firing parameters (e.g., the amount of powder) are similar but not identical between the experiments for the gas effect and with the mortar targets. If the correction is inaccurate, it could cause some error in the momentum results. On the other hand, the angular momentum is free from the gas effect.

\section{Results}

The momentum vector of the post-impact targets is expressed in the two components shown in Fig. 1, that is, the normal and tangential components at the impact point. We divide the momentum by the projectiles' initial momentum in each component, and define the efficiencies of the momentum transfer as follows;

$$
\begin{aligned}
& \eta=\frac{M^{\prime} V_{n}^{\prime}}{m v \cos \theta}, \\
& \zeta=\frac{M^{\prime} V_{t}^{\prime}}{m v \sin \theta},
\end{aligned}
$$

where $m$ and $M^{\prime}$ are respectively the mass of the projectiles and the post-impact targets. The velocities of the projectiles and the post-impact targets, $V^{\prime}$, are measured in a coordinate system where the target is initially at rest. The subscripts $n$ and $t$ denote respectively the normal and tangential components.

The efficiency of angular momentum transfer is defined as

$$
\zeta_{L}=\frac{I^{\prime} \Omega^{\prime}}{a m v \sin \theta},
$$

where $I^{\prime}$ and $\Omega^{\prime}$ are the moment of inertia and the angular velocity of the post-impact targets. It should be noted that the denominator of Eq. (4) represents the angular momentum 
Table 1. Summary of experiments-I.

\begin{tabular}{|c|c|c|c|c|c|c|c|c|}
\hline Shot No. & $\begin{array}{c}v \\
(\mathrm{~km} / \mathrm{s})\end{array}$ & $\begin{array}{c}\theta \\
\text { (degree) }\end{array}$ & $\begin{array}{c}d \\
(\mathrm{~mm})\end{array}$ & $\begin{array}{l}M \\
(\mathrm{~g})\end{array}$ & $\begin{array}{l}\Delta M \\
\text { (g) }\end{array}$ & $\eta$ & $\zeta$ & $\zeta_{L}$ \\
\hline L-49 & 3.45 & $42 \pm 3$ & 90 & 768.3 & 82.7 & $1.95 \pm 0.10$ & $-0.046 \pm 0.124$ & $0.212 \pm 0.013$ \\
\hline $\mathrm{L}-50$ & 3.45 & $42 \pm 3$ & 120 & 1799.4 & 141.6 & $2.01 \pm 0.11$ & $-0.110 \pm 0.144$ & $0.209 \pm 0.014$ \\
\hline L-52 & 3.21 & $42 \pm 2.5$ & 150 & 3298.9 & 27.3 & $1.31 \pm 0.16$ & $0.495 \pm 0.196$ & $0.230 \pm 0.027$ \\
\hline $\mathrm{L}-53$ & 3.30 & $33 \pm 4$ & 64 & 238.9 & 63.7 & $1.31 \pm 0.09$ & $-0.501 \pm 0.211$ & $0.085 \pm 0.034$ \\
\hline $\mathrm{L}-54$ & 1.98 & $34 \pm 3$ & 63 & 232.2 & 29.0 & $1.42 \pm 0.07$ & $-0.091 \pm 0.125$ & $0.272 \pm 0.031$ \\
\hline L-55 & 1.95 & $30 \pm 2$ & 93 & 806.5 & 32.1 & $1.58 \pm 0.07$ & $0.084 \pm 0.116$ & $0.316 \pm 0.024$ \\
\hline $\mathrm{L}-81$ & 3.29 & $63 \pm 2$ & 150 & 3378.0 & 22.0 & $1.64 \pm 0.32$ & $-0.022 \pm 0.111$ & $0.126 \pm 0.014$ \\
\hline L-82 & $3.31^{*}$ & $55 \pm 5$ & 122 & 1778.1 & 27.3 & $1.35 \pm 0.18$ & $0.113 \pm 0.113$ & $0.163 \pm 0.017$ \\
\hline L-83 & 3.52 & $60 \pm 5$ & 93 & 819.6 & 17.0 & $1.04 \pm 0.16$ & $0.052 \pm 0.062$ & $0.095 \pm 0.008$ \\
\hline L-84 & 3.26 & $60 \pm 10$ & 62 & 238.0 & 33.8 & $1.34 \pm 0.39$ & $-0.001 \pm 0.140$ & $0.093 \pm 0.010$ \\
\hline
\end{tabular}

$v$ : impact velocity; $\theta$ : incident angle of projectile measured from surface normal; $d$ : target's initial diameter; $M$ : target's initial mass; $\Delta M$ : target mass loss. $\eta, \zeta$ and $\zeta_{L}$ are with standard errors. *: the velocity was estimated from gun-firing parameters.

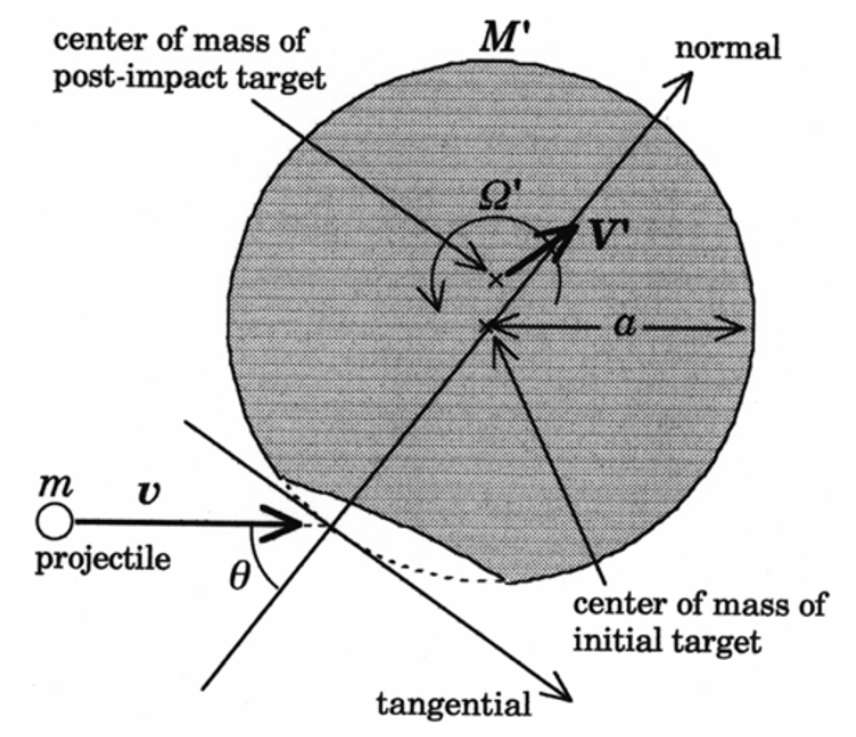

Fig. 1. Schematic illustration of a collision of a projectile with a spherical target. Normal and tangential axes are drawn through an impact point. The normal-tangential plane contains projectile's incoming trajectory and the center of mass of the target. The target initially at rest rotates with the angular velocity, $\Omega^{\prime}$, and moves with the velocity, $\boldsymbol{V}^{\prime}$, after the impact.

of the projectiles around the center of mass of the pre-impact targets while the numerator represents the angular momentum around the center of mass of the post-impact targets. The efficiencies thus defined are listed in Table 1 for all shots.

The shape of the post-impact targets was approximated by a sphere a portion of which was cut off by a plane (Fig. 2). The size of the portion was determined from the targets' mass lost by impact. The moment of inertia around an axis that is parallel to the plane and passes through the center of mass of the post-impact targets was calculated for $I^{\prime}$ in Eq. (4).

We examined the relationship between these efficiencies and the curvature radius of the targets. Target diameters vary from 90 to $150 \mathrm{~mm}$ in the three experiments at $v \approx 3.4 \mathrm{~km} / \mathrm{s}$ and $\theta=42^{\circ}$ (Shot Nos. 49, 50, and 52). They vary from 62 to $150 \mathrm{~mm}$ in the four experiments at $v \approx 3.3 \mathrm{~km} / \mathrm{s}$ and $\theta \approx 60^{\circ}$ (Shot Nos. 81-84). No clear correlation was found between the diameter and the efficiencies (Table 1) for each set of experiments at the fixed impact velocity and incident angle. However, the number of experiments would not be enough to conclude the negligible effect of the curvature radius on the efficiencies.

We plot $\zeta_{L}$ against the incident angle in Fig. 3. Filled and open symbols represent the results at the impact velocity of about 2.0 and $3.3 \mathrm{~km} / \mathrm{s}$, respectively. We took the errors of observed angular velocity, impact velocity, incident angle, and target radius into account to calculate standard errors for $\zeta_{L}$. The error of $I^{\prime}$ is not considered. The smallest value of $\zeta_{L}$ at $\theta=30^{\circ}$ corresponds to the experiment of the largest specific kinetic energy and the largest fraction of target's mass lost by impact in all our shots (Shot No. L-53). The crater is so large that it occupies the whole area surrounded by the GFRP tape. The tape would affect the ejection of target material. The other plots show clear negative correlation between $\zeta_{L}$ and the incident angle. Concordance of results at the two impact velocities indicates weak dependence of $\zeta_{L}$ on the impact velocity. The weak dependence is also shown by Shirono et al. (1993) in the velocity range of 2 to $4 \mathrm{~km} / \mathrm{s}$. They obtained the efficiency of $0.2-0.3$ at $\theta=30^{\circ}$, which agrees to our results. The negative correlation is also shown in the low-velocity impact experiments (Yanagisawa et al., 1991), and could be a general tendency in oblique impact.

Impulse vectors that act over crater surface of a postimpact target are represented by an impulse vector, $\boldsymbol{\Phi}$, that acts at a point (Fig. 4). We refer to them as the "equivalent impulse" and the "equivalent point of action", respectively. The measurement of both the momentum vector and the angular momentum enables us estimating the location of the equivalent point. The angular momentum vector is perpendicular to the projected plane shown in this figure, and we 


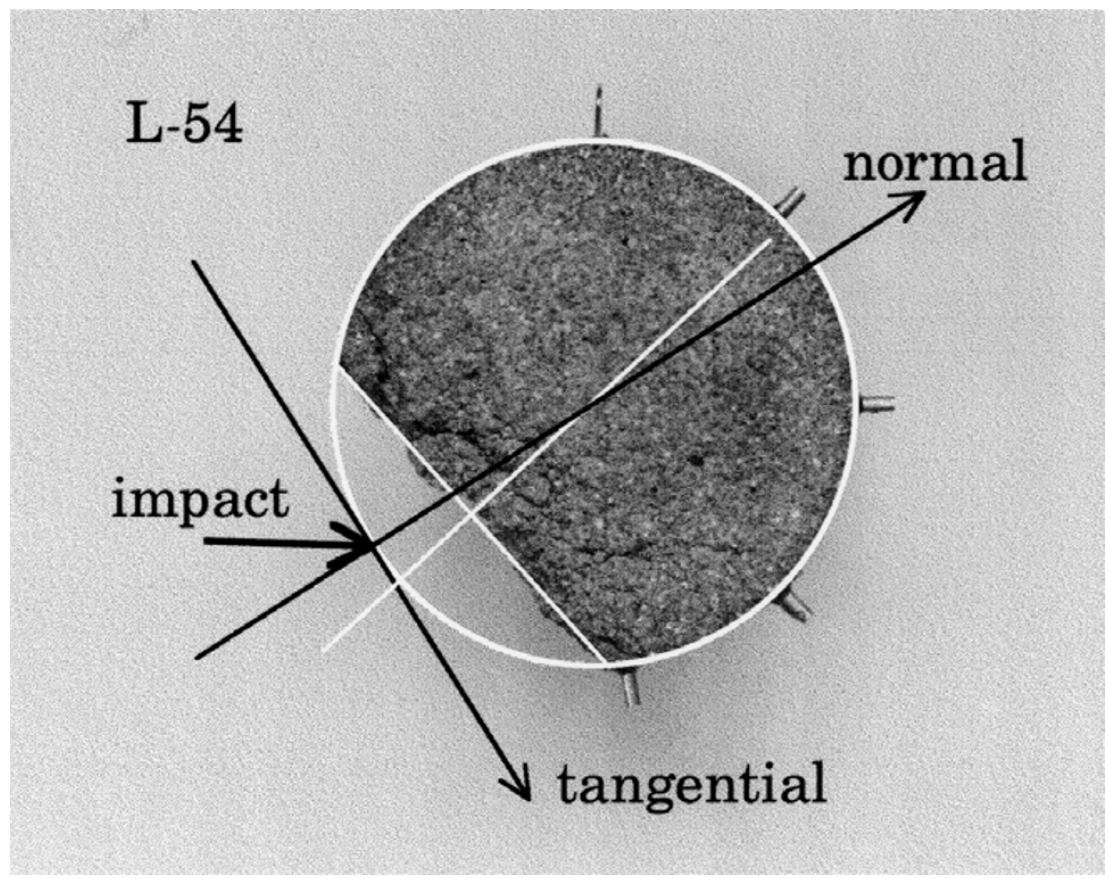

(a)

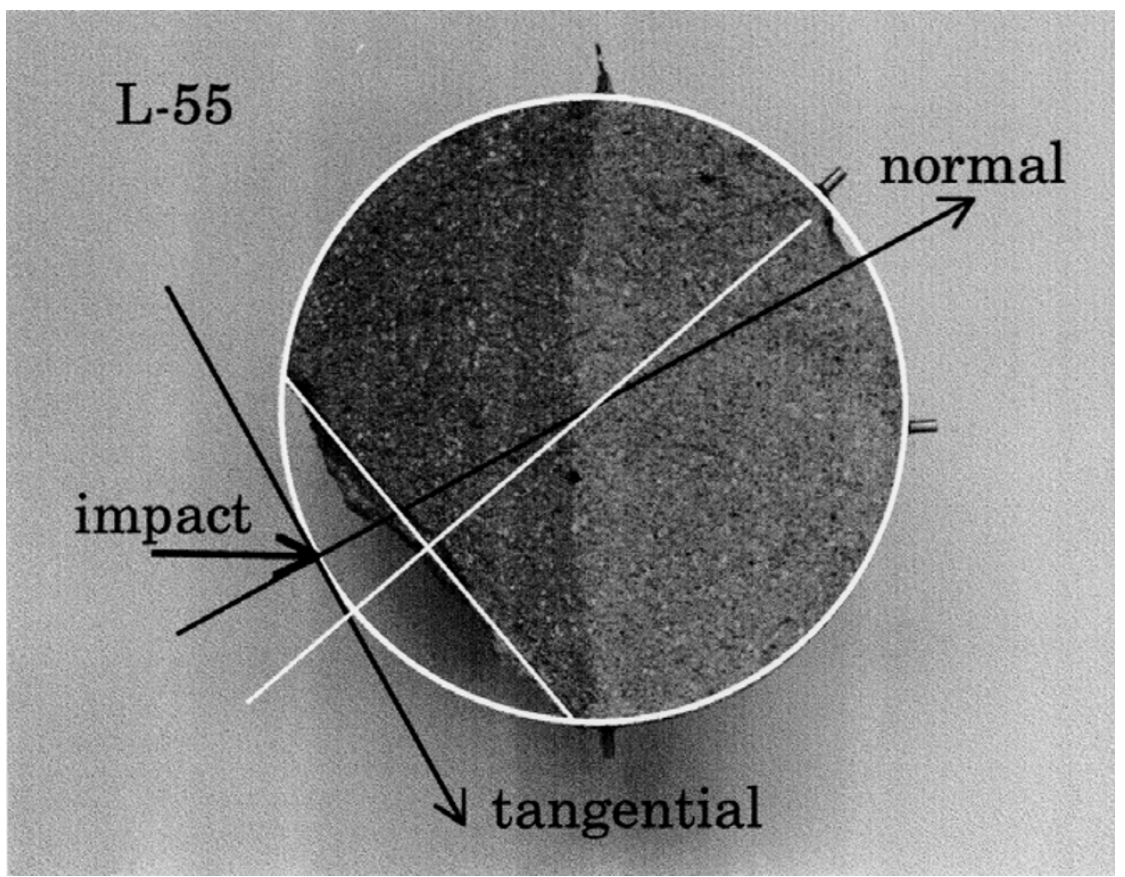

(b)

Fig. 2. Cross sections of the post-impact targets for Shot Nos. L-54 (a) and L-55 (b). The configuration of these photographs corresponds to that of Fig. 1. Impact craters are well approximated by flat surfaces (white straight line segments in the photographs). The perpendicular bisectors of the segments make some angle with the surface normals at the impact points.

take only its magnitude into account. The angular momentum around the center of mass of the initial targets is equal to the moment by the equivalent impulse as follows;

$$
\begin{gathered}
\zeta_{L} a m v_{t}-\gamma a \sin \phi \cdot \eta m v_{n}-\gamma a \cos \phi \cdot \zeta m v_{t} \\
=a^{\prime} \sin \delta \cdot \eta m v_{n}+a^{\prime} \cos \delta \cdot \zeta m v_{t} .
\end{gathered}
$$

Here, $v_{n}$ and $v_{t}$ are the two components of the impact velocity. $\gamma a$ is the distance between the center of mass before and after the impact (Fig. 4). $a^{\prime}$ is the length from the initial center to the equivalent point. The shape of the post-impact targets is approximated as described above, and their axis of symmetry makes an angle of $\phi$ with the $n$ axis. The equivalent points are displaced from the impact points by $\delta$ as shown in the figure. The first term in the left-hand side represents the rotation of 


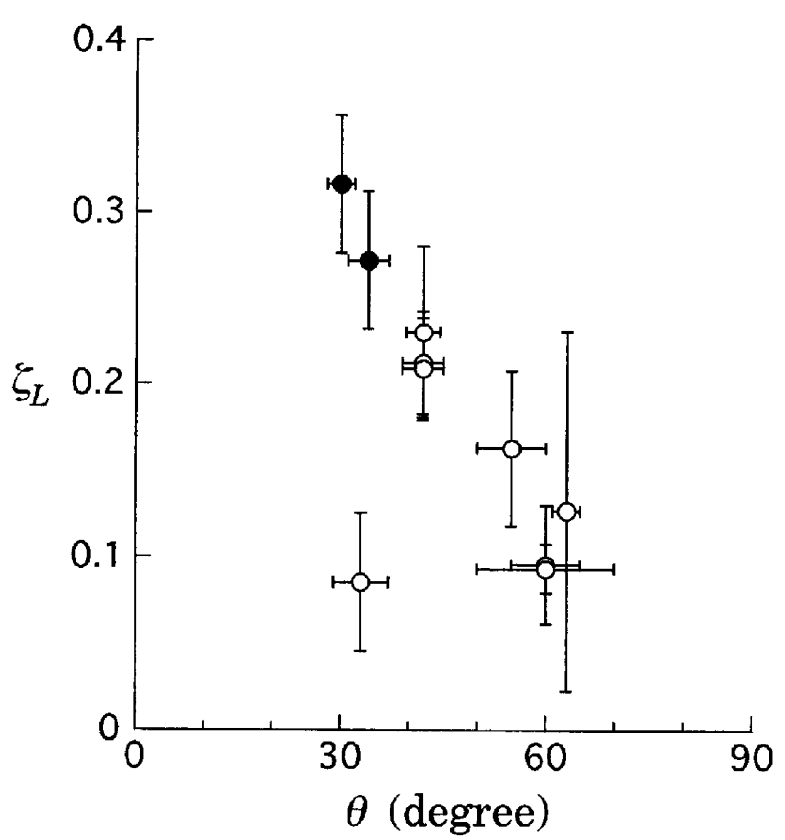

Fig. 3. $\zeta_{L}$ are plotted vs. the incident angles. Filled and open circles correspond respectively to the impact velocity of about 2.0 and $3.3 \mathrm{~km} / \mathrm{s}$. Error bars represent one- $\sigma$.

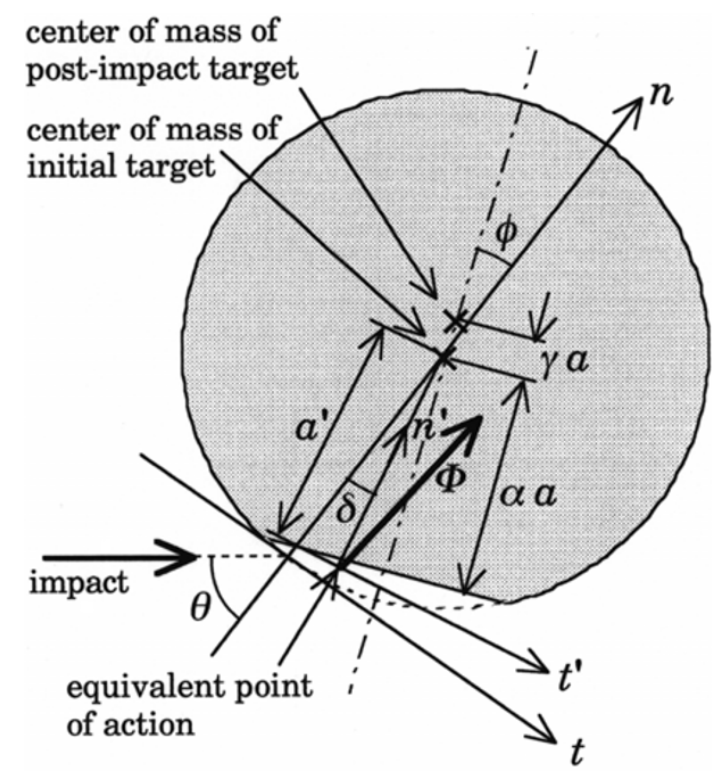

Fig. 4. Illustration shows what the parameters denote, which are used in Eq. (5). The equation is solved to find an equivalent point of action for each experiment.

the targets around the post-impact center. The second and third terms express the translational angular momentum of the post-impact targets around the initial center, where $\eta m v_{n}$ and $\zeta m v_{t}$ represent the targets' momentum. The equivalent impulse vector, $\boldsymbol{\Phi}$, is identical to the momentum vector of the target, and the moment is expressed as in the right hand side. We solve the equation for $\delta$ giving a free parameter of $a^{\prime}$.

$\alpha$ (Fig. 4) and $\gamma$ are calculated from the mass loss of the tar-

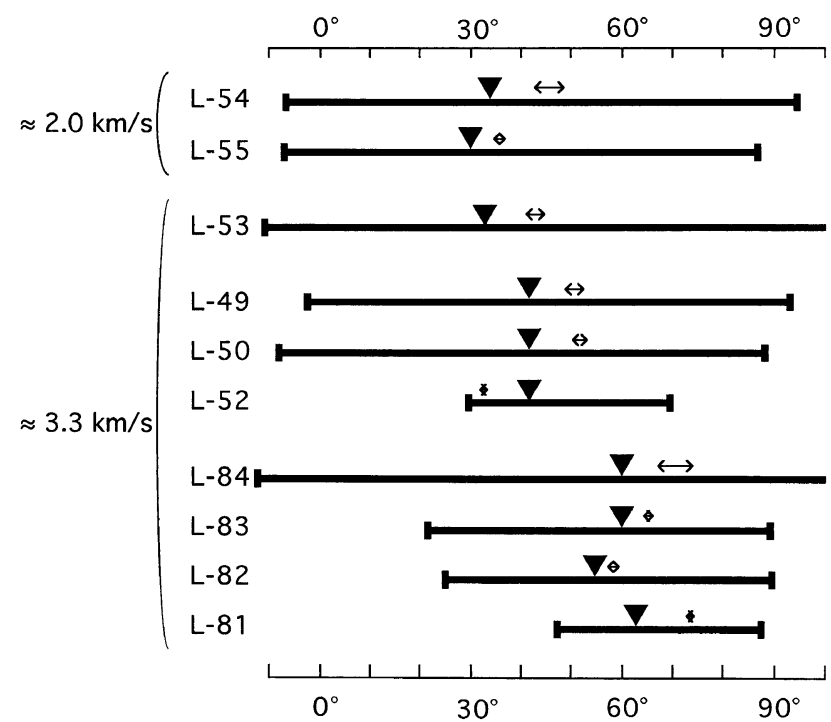

(a)

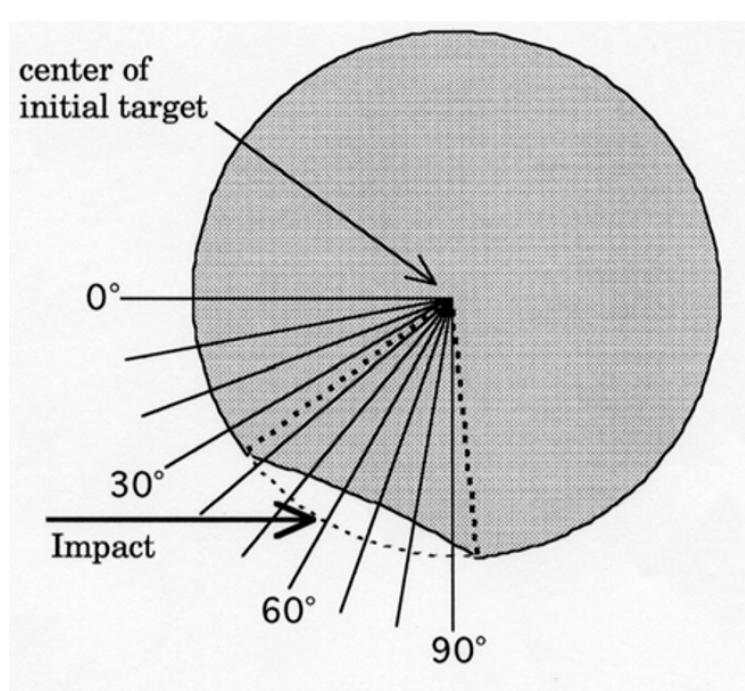

(b)

Fig. 5. (a) Locations of impact point (triangle), crater (thick line with bars), and the equivalent point of action (line with arrows) for each experiment. Locations are measured by position angle defined in (b).

gets. We determined the axis of symmetry of the post-impact targets as the perpendicular bisector of a chord joining the uprange and down-range edges of the projected crater. Whether a piece of fragment is attached to or detached from the targets occurs by chance at the crater edges, and the fragments are sizable in many cases. The error of $\phi$ must therefore be fairly large. $\alpha, \gamma$, and $\phi$ are summarized in Table 2. We assumed the range of the free parameter $a^{\prime}$ between $a$ and $\alpha a . \delta$ for the two extremes of $a^{\prime}$ are listed in Table 2 and shown thematically in Fig. 5(a). Position angle in the figure is measured as illustrated in Fig. 5(b). Lines with arrows are the ranges of $\delta$ thus derived. The impact points are plotted by triangles. Thick lines represent craters, and the bars at their end correspond to the edge of the craters. In spite of the uncertainties on the approximation for the targets' shape and the derivation of $\phi$, the equivalent points of action are 
Table 2. Summary of experiments-II.

\begin{tabular}{cccccccc}
\hline Shot No. & $\begin{array}{c}\phi \\
\text { (degree) }\end{array}$ & $\alpha$ & $\gamma$ & $\begin{array}{c}\delta \\
\left(\text { for } a^{\prime}=a\right)\end{array}$ & $\begin{array}{c}\delta \\
\left(\text { for } a^{\prime}=\alpha a\right)\end{array}$ & $\Phi / m v$ & $\psi$ \\
\hline L-49 & 3 & 0.59 & 0.089 & 7 & 10 & $1.45 \pm 0.07$ & 7 \\
L-50 & -2 & 0.66 & 0.066 & 9 & 12 & $1.50 \pm 0.08$ & 7 \\
L-52 & 8 & 0.89 & 0.0078 & -10 & -9 & $1.03 \pm 0.12$ & -4 \\
L-53 & 35 & 0.32 & 0.21 & 12 & 8 & $1.14 \pm 0.08$ & 9 \\
L-54 & 10 & 0.56 & 0.10 & 9 & 14 & $1.18 \pm 0.06$ & 7 \\
L-55 & 10 & 0.76 & 0.035 & 5 & 6 & $1.37 \pm 0.06$ & 9 \\
L-81 & 4 & 0.91 & 0.0062 & 10 & 11 & $0.746 \pm 0.145$ & 11 \\
L-82 & 3 & 0.85 & 0.014 & 3 & 5 & $0.777 \pm 0.103$ & 10 \\
L-83 & -4 & 0.83 & 0.019 & 4 & 6 & $0.524 \pm 0.080$ & 11 \\
L-84 & -4 & 0.53 & 0.11 & 7 & 14 & $0.669 \pm 0.195$ & 9 \\
\hline
\end{tabular}

located downrange of the impact point for all experiments except Shot No. L-52. The deviation of the target's shape from sphere may lead to this strange result.

We show the impulse vector in the coordinate system where its origin is the equivalent point and the normal $\left(n^{\prime}\right)$ and tangential $\left(t^{\prime}\right)$ axes are as shown in Fig. 4. The $n^{\prime}$ axis is directed from the equivalent point to the initial center of the targets, and the $t^{\prime}$ axis makes right angle with it in the projected plane. For the conversion from the $n-t$ system to the $n^{\prime}-t^{\prime}$ system, we used the mean of $\delta$ for the two extremes of $a^{\prime}$. The vectors are normalized to the magnitude of the projectiles' momentum (Fig. 6). The magnitude of the impulse and the angle between the vector and the normal axis, $\psi$, are summarized in Table 2. $\psi$ is small and in the ranges of $7^{\circ}-$ $11^{\circ}$ except Shot No. L-52. The $n^{\prime}$ axis is normal to the local surface at "epicenter" just above the equivalent point. The equivalent impulse that acts on non-spherical targets could be extrapolated from these results as follows; the impulse would be nearly normal to the local surface at the epicenter that is located downrange of the impact points.

\section{Discussion}

The angular momentum, $\Delta L$, that a spherical asteroid obtains through successive non-disruptive collisions accumulates quadratically as

$$
(\Delta L)^{2}=\sum_{i}\left(\zeta_{L}\left(\theta_{i}\right) m_{i} v_{i} a \sin \theta_{i}\right)^{2}
$$

where $\zeta_{L}$ is a function of $\theta$ as shown in the previous section. We divide $(\Delta L)^{2}$ by the sum of the squares of the angular momentum of the projectiles' translational motion, and derive a constant that can be used as the efficiency for the random walk process.

$$
\left(\zeta_{R}\right)^{2}=\frac{(\Delta L)^{2}}{\sum_{i}\left(m_{i} v_{i} a \sin \theta_{i}\right)^{2}} .
$$

For infinite number of random collisions, projectiles' mass and velocity in these equations can be fixed. The summations

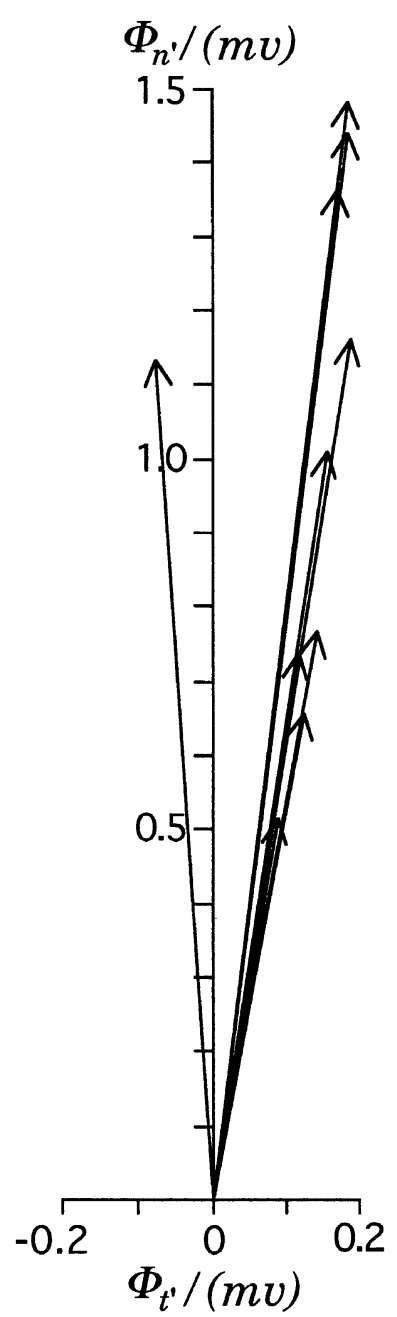

Fig. 6. Impulse vectors normalized to the magnitude of projectiles' momentum. $n^{\prime}$ and $t^{\prime}$ components are normal and tangential to the surface at the "epicenter" just above the equivalent point of action. 
Table 3. Parameters for estimating the precession angle of 1989ML.

\begin{tabular}{lcl}
\hline \multicolumn{1}{c}{ Parameters } & Variables & \multicolumn{1}{c}{ Values } \\
\hline Shear modulus of the asteroid & $\mu$ & $6.9 \times 10^{10} \mathrm{dyne} / \mathrm{cm}^{2}$ \\
Quality factor & $Q$ & 100 \\
Density & $\rho$ & $1.9 \mathrm{~g} / \mathrm{cm}^{3}$ \\
Shape factor & $K_{3}^{2}$ & 0.01 \\
Radius & $r$ & $5 \times 10^{4} \mathrm{~cm}$ \\
Minimum specific energy for shattering & $Q_{S}^{*}$ & $5 \times 10^{6} \mathrm{erg} / \mathrm{g}$ \\
Impact velocity & $v$ & $5 \times 10^{5} \mathrm{~cm} / \mathrm{s}$ \\
Coefficient for $n(m)$ & $C$ & $1.7 \times 10^{-22} \mathrm{in} \mathrm{cgs} \mathrm{unit}$ \\
Exponent for $n(m)$ & $q$ & 1.837 \\
Angular momentum transfer efficiency & $\zeta_{R}$ & 0.17 \\
\hline
\end{tabular}

Table 4. Predicted precession angles for spherical 1989ML.

\begin{tabular}{lccc}
\hline Rotation period (hours) & 5 & 10 & 20 \\
Precession damping time (years) & $1.1 \times 10^{8}$ & $9.4 \times 10^{8}$ & $7.0 \times 10^{9}$ \\
Collisional life-time (years) & $3.0 \times 10^{7}$ & $3.0 \times 10^{7}$ & $3.0 \times 10^{7}$ \\
Precession angle (degrees) & 14 & 28 & 55 \\
\hline
\end{tabular}

can be replaced by the integration over the asteroidal surface and over the impact direction at each infinitesimal surface element. The integration over the surface is not necessary for spherical target because the contributions of the surface elements are equivalent to one another. We then obtain

$$
\left(\zeta_{R}\right)^{2}=\frac{(m v a)^{2} \int_{0}^{2 \pi} \int_{0}^{\frac{\pi}{2}}\left(\zeta_{L}(\theta) \sin \theta\right)^{2} \cdot \sin \theta \cdot \cos \theta \cdot d \theta \cdot d \phi}{(m v a)^{2} \int_{0}^{2 \pi} \int_{0}^{\frac{\pi}{2}}(\sin \theta)^{2} \cdot \sin \theta \cdot \cos \theta \cdot d \theta \cdot d \phi} .
$$

We best-fit

$$
\zeta_{L}(\theta)=\zeta_{L}^{0}(\cos \theta)^{2}
$$

to the plots in Fig. 3 except an anomalous one for Shot No. L-53 and obtained $\zeta_{L}^{0}=0.41$. We adopted this function just to make the analytical integration easy. $\zeta_{R}$ is then 0.17 from Eq. (8). This value should be used for the efficiency in the formula expressing the asteroidal rotational evolution (equation (10) in Harris, 1979) or precession angle (equation (40) in B\&S1973) as long as asteroids are spherical in shape, their material properties are similar to solid mortar, and the extrapolation from laboratory experiments to asteroids' size is valid.

We apply this efficiency to B\&S1973 and estimate the precession angle of $1989 \mathrm{ML}$. $V-R-I$ band colorimetric observations suggest the asteroid to be C-type (Abe et al., 1999; Hicks et al., 1999; Ishibashi et al., 1999). Its absolute magnitude in $V$ band of $H=18.85 \pm 0.04$ indicates its size to be about $1 \mathrm{~km}$ or less (Hicks et al., 1999). The gravity would not play important roles in its collisional evolution. The results of laboratory experiments, which can not simulate gravity, therefore could be adopted reliably.
At first, we calculate the time scale for the damping of the precession and collisional lifetime. The equation (24) in B\&S1973 expresses the damping time scale for asteroid of $\omega$ in rotational angular velocity as

$$
\tau=\frac{\mu Q}{\rho K_{3}^{2} r^{2} \omega^{3}}
$$

Parameters in this and the following equations are tabulated in Table 3 with the values used for the calculation. We assume that the physical properties of mortar and asteroidal material would be similar to each other, and adopted the density for the mortar used in the experiments. The rigidity, $\mu$, is calculated from its shear wave velocity and the density. The minimum of $Q$ values in B\&S1973 is used because they assumed solid rocks and the value would be smaller for mortar.

Observations of 1989ML by Ishibashi et al. (1999) suggest the lightcurve amplitude of 1.0 in magnitude with the rotation period of 32 hours. On the other hand, Hicks et al. (1999) shows its amplitude of 0.6 in magnitude and the rotation period of 19 hours. Abe et al. (1999) indicates the both possibilities. These observations show non-spherical shape of this asteroid. We assume however that $1989 \mathrm{ML}$ is nearly spherical in shape because the efficiency of angular momentum transfer we derived is applicable only to spherical bodies. The shape factor, $K_{3}^{2}$, is then 0.01 (B\&S1973). The rotation period of $1989 \mathrm{ML}$ is not well known as described above. We therefore calculated the damping time scales for three rotation period, 5, 10, and 20 hours (Table 4).

Harris (1994) examined the values of $\mu$ and $Q$ in Eq. (10). He then calculated the ranges of the diameter and the rotation 
period of asteroids where the damping time scale is longer than $10^{8}, 10^{9}$, and $4.5 \times 10^{9}$ years, respectively. The time scale is larger than $10^{8}$ years at $1 \mathrm{~km}$ in diameter if rotation period is more than 8 hours. His results are almost consistent with ours shown in Table 4.

The lifetime, $t_{f}$, is obtained by requiring that the probability of the collision with a body larger than $m_{1}$ is unity.

$$
\pi r^{2} v t_{f} \int_{m_{1}}^{\infty} n(m) d m=1
$$

where

$$
n(m)=C m^{-q} .
$$

$n(m)$ is the number density of the impacting bodies per unit mass range, and the values for $C$ and $q$ shown in Table 3 are from B\&S1973. We derive $m_{1}$ from the specific impact energy needed to shatter a body, $Q_{S}^{*}$.

$$
Q_{S}^{*}=\frac{\frac{1}{2} m_{1} v^{2}}{M}
$$

where $M$ is the target's initial mass. $Q_{S}^{*}$ for mortar (Fujiwara et al., 1989) was adopted. The Eqs. (11), (12), and (13) are solved for $t_{f}$ as

$$
\begin{aligned}
t_{f} & =\frac{q-1}{\pi C r^{2} v} m_{1}^{q-1} \\
& =\frac{q-1}{\pi C}\left(\frac{8}{3} \pi\right)^{q-1} r^{3 q-5} v^{1-2 q} \rho^{q-1} Q_{S}^{* q-1} .
\end{aligned}
$$

The calculated collisional lifetime is shorter than the damping time for all rotation periods (Table 4).

The damping is therefore negligible, and the precession angle, $\beta$, accumulates quadratically with time as

$$
\beta^{2}=\frac{25}{12} \frac{\zeta_{R}^{2} \pi C}{(3-q)} \frac{v^{3}}{M^{2} \omega^{2}} m_{1}^{3-q} \cdot t
$$

(equation (34) in B\&S1973). The upper limit of $\beta$ is obtained by replacing $t$ in Eq. (15) by $t_{f}$ in Eq. (14) as follows,

$$
\beta_{f}^{2}=\frac{25}{3} \frac{q-1}{3-q} \zeta_{R}^{2}\left(\frac{Q_{S}^{*}}{r v \omega}\right)^{2} .
$$

In spite of the small efficiency derived in this work compared with unity adopted in B\&S1973, fairly large angles are derived from Eq. (16) (Table 4) because of the small size of 1989ML. According to Eq. (15), the precession angle is proportional to the square root of the time when it was in the collisional environment as expressed by Eqs. (11) and (12), that is, in the asteroid belt. We do not know the age of 1989ML after its formation probably by collisional disruption of its parent body, nor the duration in the asteroid belt. 1989ML is one of the Mars crossing asteroids (MCAs). Migliorini et al. (1998) and Morbidelli and Nesvorný (1999) made numerical simulations on the orbital evolution of the asteroids that stay in the inner part of the asteroid belt but do not cross Martian orbit (simimajor axis $=2.1$ to $2.5 \mathrm{AU}$ and perihelion distance $<1.8 \mathrm{AU})$. They show that about $25 \%$ of them become MCAs in 100 million years. This result indicates that MCAs could have stayed in the asteroid belt for more than 100 million year on average. The duration when 1989ML was in the belt, then, may not be so different from its lifetime. Although the duration is one tenth of its lifetime, the precession would be detectable from ground observation if it is rotating slowly with period of about 20 hours. The detection by the imaging experiment on MUSES-C would be easy even if the period is about 5 hours or less.

The shape of 1989ML would not be spherical as noted above. The non-spherical shape would increase the shape factor, $K_{3}^{2}$ in Eq. (10), from 0.01 to about 0.05 (B\&S1973). The damping time is then reduced by a factor of five, but it is still larger than or nearly equal to the collisional lifetime. The damping therefore would not be important for the estimation of the precession angle. On the other hand, the efficiency of the angular momentum transfer could be larger for non-spherical asteroids. As shown in Fig. 6, the impulse due to collision is directed nearly perpendicular to the local surface. In other words, a collision pushes the target's surface vertically. The large perpendicular component of the impulse does not spin up spherical asteroids. However, it contributes the rotation of non-spherical asteroids Yanagisawa and Hasegawa (1999) calculated the efficiency of angular momentum transfer for ellipsoidal asteroids from the experimentally derived collisional impulse. They found that the efficiency increased importantly with the axial ratio, $a / b$ or $b / c$, where $a>b>c$ were principal radii of the ellipsoids. The precession angle is proportional to the efficiency (Eq. (16)). The precession angle of 1989ML could therefore be larger than the values discussed above.

Acknowledgments. We thank N. Shirogane, N. Nakayama and T. Suminaka who participated in the experiments. We gratefully acknowledge the invaluable help given by A. Fujiwara, T. Kadono, A. Nakamura and A. Yamori. H. Hiyagon and T. Hatakeyama kindly helped us cutting the post-impact targets. We thank S. Kanayama for the use of the high-speed video camera. We are greatly indebted to Prof. D. R. Davis and A. Nakamura for their useful comments. The experiments were supported by the Plasma Laboratory, ISAS.

\section{References}

Abe, M., I. Sato, and H. Araki, Lightcurve and color of Near-Earth-Asteroid 1989ML, Adv. Space Res., 1999 (in press).

Asphaug, E. and D. J. Scheeres, Deconstructing Castalia: Evaluating a postimpact state, Icarus, 139, 383-386, 1999.

Asphaug, E., J. M. Moore, D. Morrison, W. Benz, M. C. Nolan, and R. J. Sullivan, Mechanical and geological effects of impact cratering on Ida, Icarus, 120, 158-184, 1996.

Asphaug, E., S. J. Ostro, R. S. Hudson, D. J. Scheeres, and W. Benz, Disruption of kilometre-sized asteroids by energetic collisions, Nature, 393, 437-440, 1998

Binzel, R. P., Is 1220 Crocus a precessing, binary asteroid?, Icarus, 63, 99-108, 1985

Binzel, R. P., 1991 Urey prize lecture: Physical evolution in the solar system-Present observations as a key to the past, Icarus, 100, 274-287, 1992.

Burns, J. A. and V. S. Safronov, Asteroid nutation angles, Mon. Not. R. Astr. Soc., 165, 403-411, 1973.

Davis, D. R. and S. J. Weidenschilling, Catastrophic disruption and momentum transfer in high-speed impacts, Lunar Planet. Sci., XIII, 142-143, 1982.

Davis, D. R., S. J. Weidenschilling, P. Farinella, P. Paolicchi, and R. P. Binzel, Asteroid collisional history: Effects on sizes and spins, in Asteroids II edited by R. P. Binzel, T. Gehrels, and M. S. Matthews, pp. 805-826, Univ. Arizona Press, Tucson, 1989.

Dobrovolskis, A. R. and J. A. Burns, Angular momentum drain: A mechanism for despinning asteroids, Icarus, 57, 464-476, 1984

Fujiwara, A. and A. Tsukamoto, Rotation of fragments in catastrophic im- 
pact, Icarus, 48, 329-334, 1981.

Fujiwara, A., P. Cerroni, D. Davis, E. Ryan, M. Di Martino, K. Holsapple, and $\mathrm{K}$. Housen, Experiments and scaling laws for catastrophic collisions, in Asteroids II, edited by R. P. Binzel, T. Gehrels, and M. S. Matthews, pp. 240-265, Univ. Arizona Press, Tucson, 1989.

Fujiwara, A., J. Kawaguchi, T. Mukai, and T. Uesugi, Sample return mission to NEA (MUSES-C), Adv. Space Res., 1999 (in press).

Gault, D. E. and P. H. Schultz, Oblique impact: Projectile ricochet, concomitant ejecta, and momentum transfer, Meteoritics, 21, 368-369, 1986.

Harris, A. W., Asteroid rotation rates II: A theory for the collisional evolution of rotation rates, Icarus, 40, 145-153, 1979.

Harris, A. W., Tumbling asteroids, Icarus, 107, 209-211, 1994.

Hicks, M. D., B. J. Buratti, D. R. Rabinowitz, and U. Fink, Colors and lightcurve properties of MUSES-C target 1989ML, Asteroids, Comets, Meteors 1999 (abstract).

Ishibashi Y., M. Abe, Y. Takagi, I. Sato, H. Araki, S. Hasegawa, and A. Fujiwara, Ground-based observations of Nereus and 1989ML: Target candidates for Near-Earth-Asteroid missions, Asteroids, Comets, Meteors 1999 (abstract).

Migliorini, F., P. Michel, A. Morbidelli, D. Nesvorný, and V. Zappala, Origin of multikilometer Earth- and Mars-crossing asteroids: A quantitative simulation, Science, 281, 2022-2024, 1998.
Morbidelli, A. and D. Nesvorný, Numerous weak resonances drive asteroids toward terrestrial planets orbits, Icarus, 139, 295-308, 1999.

Prokof'eva, V. V., V. P. Tarashchuk, and N. N. Gor'kavy̆, Satellites of asteroids, Physics-Uspekhi, 38, 623-649, 1995.

Shirono, S., M. Tada, A. M. Nakamura, T. Kadono, A. Rivkin, and A. Fujiwara, Efficiency of linear and angular momentum transfer in oblique impact, Planet. Space Sci., 41, 687-692, 1993.

Schultz, P. H. and D. E. Gault, Momentum transfer from oblique impacts, Lunar Planet. Sci., XVII, 781-782, 1986.

Yanagisawa, M. and S. Hasegawa, Momentum transfer in oblique impacts: Implications for asteroid rotations, Icarus, 1999 (submitted).

Yanagisawa, M., J. Eluszkiewicz, and T. J. Ahrens, Angular momentum transfer in low velocity oblique impacts: Implications for asteroids, Icarus, 94, 272-282, 1991.

Yanagisawa, M., S. Hasegawa, and N. Shirogane, Momentum and angular momentum transfer in oblique impacts: Implications for asteroid rotations, Icarus, 123, 192-206, 1996.

M. Yanagisawa (e-mail: yanagi@ee.uec.ac.jp) and S. Hasegawa (e-mail: hasegawa@planeta.sci.isas.ac.jp) 